\title{
APROVEITAMENTO DO GERMOPLASMA DE Cryptomeria japonica DA ESTAÇÃO EXPERIMENTAL DE RIO NEGRO, PR, VISANDO A PRODUÇÃO DE SEMENTES
}

\author{
Mário Dobner Júnior ${ }^{1}$, Thais Cristina Vagaes², Antonio Rioyei Higa ${ }^{3}$ \\ ${ }^{1}$ Eng. Florestal, M.Sc., Doutorando em Engenharia Florestal, UFPR, Curitiba, PR, Brasil - dobnerjr@ gmail.com \\ ${ }^{2}$ Eng $^{\mathrm{a}}$. Florestal, M.Sc., Curitiba, PR, Brasil - thaiscvagaes@yahoo.com.br \\ ${ }^{3}$ Eng. Florestal, Dr., Depto. de Ciências Florestais, UFPR, Curitiba, PR, Brasil - higa @ufpr.br
}

Recebido para publicação: 21/11/2011 - Aceito para publicação: 25/07/2013

\begin{abstract}
Resumo
Cryptomeria japonica é originária da região temperada do Japão, onde é amplamente plantada em função da alta durabilidade e trabalhabilidade da madeira. O objetivo do presente trabalho foi avaliar o desempenho de procedências japonesas de C. japonica, implantadas em 1980 na Estação Experimental da Universidade Federal do Paraná, município de Rio Negro, Paraná. Realizou-se levantamento dendrométrico em setembro de 2011, o qual serviu de base para as análises estatísticas e genéticas. A produtividade observada aos 31 anos variou de $9,3 \mathrm{~m}^{3} \cdot \mathrm{ha}^{-1} \cdot$ ano $^{-1}$ na testemunha (Camanducaia), até 22,2 $\mathrm{m}^{3} \cdot \mathrm{ha}^{-1} \cdot \mathrm{ano}^{-1}$ na procedência Miyagi. A partir dos resultados, foram propostas estratégias de melhoramento genético para a criação de uma Área de Produção de Sementes (APS) e um Pomar Clonal de Sementes (PCS), bem como a seleção de indivíduos superiores para reprodução assexuada. Para tal, considerou-se uma intensidade de seleção que mantivesse ao máximo a variabilidade genética entre procedências, mas explorasse a variação dentro de cada procedência. As sementes produzidas terão um ganho no crescimento diamétrico estimado em 9,2\% para a APS (curto prazo) e de $14 \%$ para o PCS (médio prazo).
\end{abstract}

Palavras-chave: Melhoramento florestal; área de produção de sementes; pomar clonal de sementes.

\begin{abstract}
A proposal for the existing germplasm of Cryptomeria japonica (L.F.) Don. at the Experimental Station of Rio Negro, PR, aiming at the production of genetic improved seeds. Cryptomeria japonica is indigenous to the temperate region of Japan where it is widely planted, due to its wood quality, high durability and workability. The aim of this study was to evaluate the performance of Japanese provenances of $C$. japonica, established in 1980 at Experimental Station of Parana Federal University, municipality of Rio Negro, Parana State. Dendrometric measurements were carried out in September 2011, which was the basis for statistical and genetic analysis. The productivity at age 31 years ranged from $9.3 \mathrm{~m}^{3} \cdot \mathrm{ha}^{-1}$.year ${ }^{-1}$ (control), up to $22.2 \mathrm{~m}^{3} \cdot \mathrm{ha}^{-1}$.year ${ }^{-1}$ (Miyagi). A breeding strategy was proposed based on a seed production area (SPA) and on a clonal seed orchard (CSO). Moreover superior individuals for vegetative reproduction were also selected. The selection intensity was suggested considering the maintenance of expressive genetic variability among provenances, but exploring the variation within each provenance. The estimated genetic gain for diameter growth was $9.2 \%$ for the SPA (short term) and $14 \%$ for the CSO (medium term).

Keywords: Forest breeding; seed production area; clonal seed orchard.
\end{abstract}

\section{INTRODUÇÃO}

Uma das coníferas comerciais mais importantes na Ásia (TAKATA; TERAOKA, 2002; SHYUR et al., 2008), Cryptomeria japonica é originária da região temperada do Japão, onde é conhecida como 'Sugi' (CARPANEZZI; CARVALHO, 1988) e amplamente plantada (YAMASHITA et al., 2004; ZHU et al., 2005), principalmente em regiões montanhosas (TAKATA; TERAOKA, 2002), ocupando, aproximadamente, $45 \%$ das plantações florestais naquele país, de um total de 10 milhões de hectares (OHBA, 1993). Na área de distribuição natural, a espécie ocorre naturalmente entre 600 e 1.800 metros 
de altitude, em clima caracterizado por invernos frios, com ocorrência de neve e verões moderadamente quentes (CARPANEZZI; CARVALHO, 1988).

No Brasil, possui crescimento ótimo em locais de temperatura entre 12 e $14{ }^{\circ} \mathrm{C}$, com pluviosidade média anual acima de $2.000 \mathrm{~mm}$ (ALVES et al., 1984). Possui porte elevado, comumente com 20 a 30 metros de altura (até 50 metros na região de origem), copa cônica, tronco reto, envolvido por uma casca acastanhada e fibrosa (FABIÃO, 1987).

Santos et al. (2000) comentam que $C$. japonica possui grande potencial para plantios nas regiões altas e frias do Brasil, destacando-se pelo seu rápido crescimento, boa adaptação ao clima e solo do Sul do país e boa qualidade da madeira. A Empresa Brasileira de Pesquisa Agropecuária (EMBRAPA) (1988) recomenda plantios comerciais em locais acima de $600 \mathrm{~m}$, em solos profundos e férteis, com bom suprimento de água durante todo o ano. Shimizu e Maiochi (2007) confirmam a sensibilidade da espécie à qualidade do sítio. Segundo EMBRAPA (1988), suas exigências edáficas são equivalentes ou superiores às do pinheiro-do-paraná, fato este confirmado em seu local de origem por Yamashita et al. (2004), que relatam ser um espécie plantada apenas nos sítios mais férteis em função das suas exigências.

A madeira, amplamente utilizada no Japão (ZHU et al., 2005), é leve, com massa específica aparente entre 0,25 e $0,35 \mathrm{~g} . \mathrm{cm}^{-3}$, e não apresenta grandes problemas de contração, sendo considerada uma madeira de alta durabilidade e fácil de ser trabalhada (GURGEL FILHO, 1964; CARVALHO, 2001), podendo ser utilizada para serrados em geral e painéis compensados (SANTOS et al., 2000; GÉRARD et al., 2003). Além disso, é uma madeira estável, qualidade importante para processamento mecânico e para aplicações mais nobres, como movelaria (PEREIRA et al., 2003).

A espécie foi introduzida no Brasil pela Companhia Melhoramentos e tem sido plantada há décadas, principalmente no estado de São Paulo (750 a 1000 m a.n.m.) e no sul do estado de Minas Gerais (1.500 m a.n.m.) (CARPANEZZI; CARVALHO, 1988).

Para Carvalho (2001), C. japonica é umas das espécies arbóreas alternativas para plantios florestais no Brasil, apresentando incremento médio anual (IMA) em volume sólido com casca entre 14 e $45 \mathrm{~m}^{3} \cdot \mathrm{ha}^{-1} \cdot$ ano $^{-1}$.

Uma estratégia para o aproveitamento do potencial dessa espécie seria desenvolver um programa de melhoramento genético para produção de sementes, a partir de procedências e indivíduos selecionados dentro de populações-base, utilizando-se a variabilidade existente naturalmente dentro das populações e entre os indivíduos. Essa metodologia de trabalho é citada por Golle et al. (2009), sendo relatada como modelo padrão para o melhoramento de espécies florestais.

De forma semelhante e reconhecendo a importância econômica da espécie na Região Autônoma dos Açores, $C$. japonica foi incluída no Programa de Melhoramento Florestal Nacional (ALMEIDA et al., 2005).

Para recombinação do material genético selecionado, bem como para dar continuidade ao programa de melhoramento, utilizam-se povoamentos denominados áreas de coleta (ACS) e áreas de produção de sementes (APS), ou ainda pomares estabelecidos com o propósito específico de produzir sementes.

Segundo Lerner (1977), o propósito básico da seleção é possibilitar o agrupamento e cruzamento dos indivíduos selecionados e o aumento, a cada geração, do número de indivíduos portadores de características desejáveis. A magnitude desse aumento ou o progresso a ser alcançado com a seleção é, principalmente, função da grandeza e da natureza da variabilidade natural existente na população e da intensidade com que a mesma está sendo explorada (VENCOVSKY, 1978).

Os valores genéticos dos candidatos à seleção para caracteres específicos são variáveis aleatórias que não podem ser conhecidas exatamente. Entretanto, valores fenotípicos são utilizados visando à estimação de valores genéticos. Em programas de melhoramento, o problema principal consiste em se adotarem valores fenotípicos que melhor estimem os valores genéticos verdadeiros (RESENDE; HIGA, 1994).

Alguns estudos para determinar a herdabilidade no sentido restrito para a espécie em questão encontraram valores de 0,2 a 0,3 para a variável Diâmetro à Altura do Peito (DAP) (SAKAI; MUKAIDE, 1966; KURINOBU et al., 2000), e de 0,2 a 0,4 para a variável altura (SAKAI; MUKAIDE, 1966; OHBA et al., 1970).

Segundo Takata e Teraoka (2002), muitos cultivares foram desenvolvidos no Japão para utilização em reflorestamentos, com o objetivo de produzir madeira.

Considerando o exposto, a espécie apresenta favorável perspectiva como fonte alternativa de madeira de qualidade para a região Sul do Brasil. Para que seja possível utilizar todo o potencial de crescimento, é necessário estabelecer um programa de melhoramento. Em função do longo tempo 
necessário, um programa de melhoramento florestal deve considerar, além do seu objetivo principal, localizado no futuro (médio prazo), ações imediatas (curto prazo) que possibilitem a utilização de material superior à população-base imediatamente.

O trabalho teve como objetivo avaliar o desempenho de diferentes procedências japonesas e, a partir do mesmo, propor estratégias de melhoramento genético, com a formação de áreas de produção de sementes (curto e médio prazo) e a seleção de indivíduos para reprodução assexuada (curto prazo). As diferenças no potencial de produção de madeira entre as procedências foram testadas com o objetivo de identificar as mais produtivas. A estratégia foi definida de forma a conservar a presente população ex situ. Para tal, propõe-se uma intensidade de seleção que preserve a variabilidade genética entre procedências, mas explore a variação dentro de cada procedência.

\section{MATERIAL E MÉTODOS}

O teste de procedência foi plantado na Estação Experimental de Rio Negro, localizada na bacia do rio Passa Três, afluente do rio Negro. Está compreendida no Primeiro Planalto Paranaense, $847 \mathrm{~m}$ acima do nível do mar. O clima é do tipo Cfa, com precipitação anual média de $1.271 \mathrm{~mm}$ e temperatura anual média de $16,4^{\circ} \mathrm{C}$ (EMBRAPA, 1986). A ocorrência de geadas no inverno é comum. Os solos da estação se dividem em dois grandes grupos, os latossolos arenosos e os solos hidromórficos.

A vegetação original da área era a Floresta Ombrófila Mista, com presença de araucárias e imbuias. A ocupação humana anterior praticamente eliminou toda a cobertura florestal nas áreas agriculturáveis e nas várzeas não sujeitas a inundações periódicas. Quando a Universidade Federal do Paraná UFPR assumiu, predominava a vegetação campestre.

O teste de procedência de $C$. japonica plantado entre fevereiro e abril de 1980 foi estabelecido com sementes de cinco origens japonesas. Foi incluída, também, uma testemunha brasileira (coletada em Camanducaia, MG), constituída de regenerações naturais da espécie um povoamento comercial no sul do estado de Minas Gerais, de origem desconhecida (Tabela 1).

Tabela 1. Dados sobre as procedências japonesas e a testemunha plantadas na EE Rio Negro.

Table 1. Data about the Japanese provenances and the control planted in EE Rio Negro.

\begin{tabular}{lccccc}
\hline Procedência & $\begin{array}{c}\text { Precipitação } \\
(\mathbf{m m})\end{array}$ & $\begin{array}{c}\text { Temperatura } \\
\left({ }^{\circ} \mathbf{C}\right)\end{array}$ & $\begin{array}{c}\text { Altitude } \\
(\mathbf{m})\end{array}$ & Latitude & Longitude \\
\hline 1. Miyagi & 1.257 & 12,2 & $40-50$ & $38^{\circ} 19^{\prime} \mathrm{N}$ & $141^{\circ} 27^{\prime} \mathrm{E}$ \\
2. Toyama & 3.902 & 8,5 & $1.000-1.300$ & $36^{\circ} 33^{\prime} \mathrm{N}$ & $137^{\circ} 35^{\prime} \mathrm{E}$ \\
3. Shimane & 2.258 & 12,8 & $700-900$ & $34^{\circ} 37^{\prime} \mathrm{N}$ & $132^{\circ} 12^{\prime} \mathrm{E}$ \\
4. Akita & 1.968 & 10,7 & $300-400$ & $40^{\circ} 00^{\prime} \mathrm{N}$ & $140^{\circ} 13^{\prime} \mathrm{E}$ \\
5. Nara & 1.616 & 13,4 & 500 & $34^{\circ} 30^{\prime} \mathrm{N}$ & $136^{\circ} 00^{\prime} \mathrm{E}$ \\
6. Camanducaia & 1.744 & 19,2 & 1.200 & $22^{\circ} 45^{\prime} \mathrm{S}$ & $46^{\circ} 08^{\prime} \mathrm{W}$ \\
\hline
\end{tabular}

Fonte: adaptado de Shimizu; Maiochi (2007).

O delineamento experimental utilizado foi o de blocos completos ao acaso, com cinco repetições e vinte e cinco plantas por parcela, com espaçamento de 3,0 x 2,0 m. No entorno do experimento, foram plantadas duas linhas de bordadura com as mudas da procedência Camanducaia.

No dia 21/09/2011, realizou-se levantamento dendrométrico, com a medição de todos os DAP e 15 alturas totais em árvores distribuídas em diversos blocos e procedências.

A análise da qualidade das árvores foi realizada avaliando-se a presença de bifurcações na base e na copa das árvores. Para evitar falsas conclusões com relação à qualidade das árvores, a presença de bifurcações foi relativizada ao número de indivíduos remanescentes por procedência.

De forma a classificar as procedências considerando o real potencial de produção de madeira, criou-se a variável DAP $(\mathrm{cm})$ x Sobrevivência $(\%)$. O resultado foi um valor adimensional que expressou o ritmo de crescimento penalizado pela sobrevivência relativa das procedências.

As análises genéticas do teste de procedência foram efetuadas com o auxílio do software SELEGEN - REML/BLUP, desenvolvido para a seleção genética computadorizada no melhoramento de espécies perenes. Resende (2002a) relata que o software é fundamentado em algoritmos que maximizam a eficiência do processo seletivo, em função de diferentes situações experimentais. 
A seleção efetuada com o auxílio do SELEGEN foi baseada nos valores genético (para propagação sexuada) e genotípico (para propagação assexuada) preditos de cada indivíduo (RESENDE, 2002a), com o modelo 24 "Blocos ao acaso, teste de populações ou procedências, várias plantas por parcela" (RESENDE, 2002b).

Para os cálculos de valores efetivos populacionais, partiu-se do princípio de que os indivíduos de cada procedência não são aparentados. Segundo Resende (2002a), as populações presentes nos testes de procedência são representadas, comumente, por uma mistura de sementes de várias matrizes, não havendo assim a identificação de progênies.

\section{RESULTADOS E DISCUSSÃO}

A tabela 2 apresenta os resultados do levantamento dendrométrico realizado em setembro de 2011, 31 anos após o plantio.

Após a confirmação da homogeneidade entre as variâncias das diferentes procedências (Levene, $p=0,684)$, realizou-se análise de comparação entre as médias das procedências, detectando-se diferenças significativas $(\mathrm{F}(5,433)=6,064 ; p=0,001)$, fato este já identificado por Shimizu e Maiochi (2007) quando o teste apresentava 25 anos de idade. Verificou-se, porém, que apenas a procedência Toyama é estatisticamente inferior às demais, sendo ainda semelhante à Camanducaia.

Tabela 2. Diâmetro à altura do peito (DAP) mínimo, médio e máximo para as diferentes procedências.

Table 2. Minimum, average and maximum diameter at breast height (DBH) for the different provenances.

\begin{tabular}{|c|c|c|c|c|}
\hline \multirow{2}{*}{ Ranking } & \multicolumn{3}{|c|}{ DAP (cm) } & \multirow{2}{*}{ Procedência } \\
\hline & Mínimo & Médio & Máximo & \\
\hline $1^{\circ}$ & 8,5 & $29,4 \quad$ a & 43,1 & Nara \\
\hline $2^{\circ}$ & 12,7 & 28,4 a & 46,2 & Miyagi \\
\hline $3^{\circ}$ & 7,2 & $27,7 \quad \mathrm{a}$ & 51,9 & Shimane \\
\hline $4^{\circ}$ & 13,1 & 27,3 a & 56,9 & Akita \\
\hline $5^{\circ}$ & 15,0 & 26,5 a b & 41,5 & Camanducaia \\
\hline $6^{\circ}$ & 11,0 & $23,8 \quad b$ & 41,9 & Toyama \\
\hline
\end{tabular}

Médias seguidas das mesmas letras não diferem estatisticamente entre si, segundo o teste de Tukey, com $95 \%$ probabilidade.

Verifica-se grande variabilidade de crescimento em DAP entre as procedências e, principalmente, entre os indivíduos de uma mesma procedência. Segundo Sebbenn et al. (1994), a variabilidade genética existente em uma população é a matéria-prima básica do melhoramento, e o conhecimento de sua distribuição é relevante para a definição de estratégias de seleção em programas de melhoramento florestal.

Outra constatação é que os maiores DAP's estão presentes nas procedências com diâmetros médios intermediários, Akita $(56,9 \mathrm{~cm})$ e Shimane $(51,9 \mathrm{~cm})$. Nesta última, também com o menor DAP amostrado.

Shimizu e Maiochi (2007) analisaram o mesmo experimento com seis anos a menos, obtendo conclusões semelhantes. Em comparação à presente análise, destaca-se o maior crescimento diamétrico das procedências Shimane e Akita nos últimos 5 anos, superando a testemunha (Camanducaia) nesse período.

Para efeito de comparação, um plantio de C. japonica aos 22 anos de idade e 1.200 indivíduos.ha $^{-1}$, localizado na região central do Japão, com temperatura média anual de $14{ }^{\circ} \mathrm{C}$ e precipitação de $1.900 \mathrm{~mm}$, apresentava DAP médio de 24,6 cm (YAMASHITA et al., 2004). Embora haja uma diferença de 9 anos entre o trabalho citado acima e o presente estudo, verifica-se certa similaridade de ritmo de crescimento diamétrico entre o local de origem e o observado no teste de procedência na EE Rio Negro.

Com os pares de dados DAP x Altura, ajustou-se o modelo hipsométrico de Stoffels $\left(\mathrm{R}_{\mathrm{aj}}^{2}:\right.$ 0,726; $\left.\mathrm{S}_{\mathrm{yx}}: 0,108\right)$ :

$$
\ln (h)=1,14229+0,5899 * \ln (d a p)
$$

Com relação à sobrevivência das procedências, verificou-se grande variação entre as mesmas. Merece destaque a procedência Camanducaia, com apenas 37\% de indivíduos remanescentes no teste. 
Embora tenha sido observada grande variação entre as procedências japonesas, todas foram superiores à testemunha (Camanducaia).

Tabela 3. Sobrevivência relativa $\left(\mathrm{S}_{\%}\right)$ por procedência.

Table 3. Provenances relative survival $\left(\mathrm{S}_{\%}\right)$.

\begin{tabular}{lcc}
\hline Ranking & $\mathbf{S}_{\boldsymbol{\%}}$ & Procedência \\
\hline $1^{\circ}$ & 76 & Miyagi \\
$2^{\circ}$ & 66 & Nara \\
$3^{\circ}$ & 62 & Toyama \\
$4^{\circ}$ & 59 & Akita \\
$5^{\circ}$ & 52 & Shimane \\
$6^{\circ}$ & 37 & Camanducaia \\
\hline
\end{tabular}

A presença de bifurcações basais deve-se, provavelmente, à incidência de geada nos primeiros anos após o plantio das mudas. O fato de o plantio ter sido realizado no mês de março de 1980 pode ter intensificado tais danos. Segundo Shimizu e Maiochi (2007), as geadas do primeiro inverno danificaram 47\% dos indivíduos presentes no teste.

Com relação às bifurcações na base da árvore, verificou-se a menor intensidade relativa às plantas sobreviventes na procedência Shimane, na qual $14 \%$ dos indivíduos apresentaram o defeito, seguida das procedências Akita (23\%), Nara (27\%), Camanducaia (28\%), Miyagi (38\%) e Toyama (39\%).

A bifurcação na copa das árvores ocorreu em menor intensidade, sendo observada em 3\% dos indivíduos das procedências Toyama e Miyagi, seguidas das procedências Nara (4\%), Camanducaia (7\%), Shimane (12\%) e Akita (16\%).

A bifurcação na base é limitante para a produção de toras de qualidade; já a bifurcação na copa, embora indesejável, não acarreta problemas na utilização das toras mais valiosas das árvores, localizadas no terço inferior das mesmas.

Com o objetivo de quantificar a produção volumétrica das procedências aos 31 anos de idade do povoamento, utilizou-se a aproximação linear do modelo Schumacher-Hall, ajustado para um plantio homogêneo da mesma espécie no município de Campo Belo do Sul (informações pessoais) com 25 anos de idade $\left(\mathrm{R}_{\mathrm{aj}}^{2}: 0,993 ; \mathrm{S}_{\mathrm{yx}}: 0,066\right)$ :

$$
\ln (V)=-10,24740+1,86384 * \ln (\text { dap })+1,12332 * \ln (h)
$$

Os estoques volumétricos aos 31 anos de idade, extrapolados para um hectare, bem como o incremento médio anual (IMA) para as diferentes procedências, podem ser observados na tabela 4. Após confirmação da homogeneidade das variâncias (Levene, $p=0,670$ ), realizou-se teste de comparação de médias, detectando-se diferenças significativas $(\mathrm{F}(5,24)=6,290 ; p=0,001)$.

Tabela 4. Estoques volumétricos $\left(\mathrm{m}^{3} / \mathrm{ha}\right)$ e incremento médio anual (IMA) $\left(\mathrm{m}^{3} / \mathrm{ha}^{-a n o^{-1}}\right)$ para as diferentes procedências.

Table 4. Volumetric stock $\left(\mathrm{m}^{3} / \mathrm{ha}\right)$ and mean annual increment (MAI) $\left(\mathrm{m}^{3} / \mathrm{ha}^{-a n o^{-1}}\right)$ for the different provenances.

\begin{tabular}{|c|c|c|}
\hline Procedência & Volume $\left(\mathrm{m}^{3} \mathrm{ha}^{-1}\right)$ & IMA $\left(\mathrm{m}^{3} \mathrm{ha}^{-1} \mathrm{ano}^{-1}\right)$ \\
\hline Miyagi & $687,5 \mathrm{a}$ & 22,2 \\
\hline Nara & $657,1 \mathrm{a}$ & 21,2 \\
\hline Akita & $513,0 \mathrm{ab}$ & 16,5 \\
\hline Shimane & $470,9 \mathrm{ab}$ & 15,2 \\
\hline Toyama & $385,8 \mathrm{~b}$ & 12,4 \\
\hline Camanducaia & $289,2 \mathrm{~b}$ & 9,3 \\
\hline
\end{tabular}

Verificaram-se diferenças estatísticas agrupando-se as procedências em dois grupos em termos de estoque volumétrico. Os valores observados são compatíveis com os relatados por Carvalho (2001), 
entretanto nenhuma procedência apresentou produtividade próxima ao máximo citado pelo autor, de $45 \mathrm{~m}^{3} \cdot \mathrm{ha}^{-1} \cdot$ ano $^{-1}$.

A classificação das procedências obtida a partir da variável adimensional DAP $(\mathrm{cm})$ x Sobrevivência $(\%)$ pode ser verificado na tabela 5. Tal classificação destaca os melhores crescimentos diamétricos, porém considerando as maiores sobrevivências relativas.

Tal análise resultou em conclusões diferentes das obtidas por Shimizu e Maiochi (2007), que avaliaram o volume individual das árvores sem considerar a sobrevivência das procedências e, consequentemente, a produção total por unidade de área.

Tabela 5. Ranking final das procedências (DAP x Sobreviência).

Table 5. Final ranking for the provenances (DBH x Survival).

\begin{tabular}{lc}
\hline Ranking & Procedência \\
\hline $1^{\circ}$ & Miyagi \\
$2^{\circ}$ & Nara \\
$3^{\circ}$ & Akita \\
$4^{\circ}$ & Shimane \\
$5^{\circ}$ & Toyama \\
$6^{\circ}$ & Camanducaia \\
\hline
\end{tabular}

Verifica-se que a criação da variável adimensional (DAP x Sobrevivência) propiciou um excelente resultado, pois foi capaz de classificar as procedências na mesma ordem na qual a análise do estoque volumétrico o fez.

\section{Proposta de desbaste genético}

A formação de uma Área de Produção de Sementes (APS) melhorada geneticamente para a produção de madeira será obtida com a realização de um desbaste genético considerando o potencial produtivo dos indivíduos e procedências apresentado aos 31 anos de idade. Os resultados da análise genética do teste de procedência são apresentados na Tabela 6.

Tabela 6. Componentes de variância (REML Individual).

Table 6. Variance components (Individual REML).

\begin{tabular}{lrr} 
Vg & 3,528 & \\
Vparc & 0,144 & \\
Ve & 46,170 & \\
Vf & 49,842 & \\
$\mathrm{~h}^{2} \mathrm{~g}$ & $0,071 \quad \pm 0,036$ \\
$\mathrm{c}^{2}$ parc & 0,003 & \\
$\mathrm{~h}^{2} \mathrm{mp}$ & 0,895 & \\
Acproc & 0,946 & \\
média geral & 27,256 \\
\hline
\end{tabular}

Vg: variância genotípica entre populações; Vparc: variância ambiental entre parcelas; Ve: variância residual; Vf: variância fenotípica individual; $\mathrm{h}^{2} \mathrm{~g}$ : herdabilidade individual no sentido amplo; $\mathrm{c}^{2}$ parc: coeficiente de determinação dos efeitos da parcela; h²mp: herdabilidade da média de populações; Acproc: acurácia da seleção de populações.

Como o objetivo do programa é preservar a variabilidade genética entre as procedências, explorando a variação dentro de cada procedência, o desbaste a ser realizado prevê, primeiramente, a seleção dos quatro melhores indivíduos por parcela, de forma que todas as procedências permaneçam na APS.

A análise das procedências individualmente permitiu concluir que as procedências Akita, Camanducaia e Toyama possuem impacto genético negativo (g) na média da população atual (Tabela 7), para a variável DAP. Entretanto, como o objetivo do presente programa de melhoramento é a manutenção da variabilidade entre as procedências, todas serão mantidas após o desbaste.

Seguindo a metodologia de seleção proposta (4 indivíduos por procedência, 6 procedências e 5 repetições), dos 120 indivíduos selecionados, 19 apresentaram valor aditivo (a) negativo para a variável DAP. São árvores das procedências Camanducaia e Toyama. Para evitar o efeito genético negativo no 
potencial produtivo das sementes produzidas no futuro pomar, tais indivíduos serão desbastados, sendo mantidos outros 19 de melhor valor genético, o quinto melhor indivíduo das primeiras quatro procedências (Tabela 5).

Tabela 7. Seleção de Procedências para a variável DAP.

Table 7. Provenances selection for DBH.

\begin{tabular}{lccccc}
\hline Ordem & Procedência & $\mathbf{g}$ & $\mathbf{u + g}$ & Ganho & Nova média \\
\hline 1 & Nara & 1,98 & 29,2 & 1,98 & 29,2 \\
2 & Miyagi & 1,16 & 28,4 & 1,57 & 28,8 \\
3 & Shimane & 0,31 & 27,6 & 1,15 & 28,4 \\
4 & Akita & $-0,01$ & 27,2 & 0,86 & 28,1 \\
5 & Camanducaia & $-0,39$ & 26,9 & 0,61 & 27,9 \\
6 & Toyama & $-3,05$ & 24,2 & 0,00 & 27,3 \\
\hline
\end{tabular}

g: efeito genético predito; u+g: média genotípica.

O ganho previsto para a intensidade de seleção proposta, a ser obtido nas sementes produzidas após a realização do desbaste genético, é de $9,2 \%$.

O tamanho efetivo populacional $\left(N_{e}\right)$ da APS será de 96, valor este considerado suficiente para a formação de uma nova população (SEBBENN, 2006) e suficiente para evitar a perda de alelos favoráveis ao longo dos ciclos de melhoramento em populações pouco melhoradas (RESENDE; BARBOSA, 2005). Em outras palavras, pode-se afirmar que o objetivo de manter a variabilidade existente entre as diferentes procedências está assegurado e não há risco de depressão endogâmica, caracterizada pela perda de heterozigose (RESENDE, 2002a).

Paralelamente ao desbaste genético no teste de procedência, formando a primeira APS de C. japonica da região Sul do Brasil, as 10 melhores árvores serão clonadas, formando assim um Pomar de Sementes (PCS), com intensidade de seleção maior e, consequentemente, maior potencial de produtividade.

As árvores selecionadas para a formação do PCS são apresentadas na Tabela 8. O ganho previsto para o crescimento diamétrico obtido com a utilização das sementes produzidas no PCS é de $14 \%$. O tamanho efetivo populacional $\left(N_{e}\right)$ do PCS será de 40. Segundo Rawlings (1970) apud Resende e Barbosa (2005), um $N_{e}$ ao redor de 30 seria adequado para a maioria dos sistemas genéticos.

Tabela 8. Seleção de árvores para implantação do Pomar Clonal de Sementes (PCS).

Table 8. Tree selection for establishment of Clonal Seeds Orchard (CSO).

\begin{tabular}{lcccccccc}
\hline Ordem & Procedência & Bloco & Árvore & $\mathbf{f}$ & $\mathbf{a}$ & $\mathbf{u + a}$ & Ganho & Nova média \\
\hline 1 & Miyagi & 5 & 17 & 39,6 & 4,82 & 32,1 & 4,8 & 32,1 \\
2 & Miyagi & 1 & 2 & 36,4 & 3,01 & 30,3 & 3,9 & 31,2 \\
3 & Nara & 3 & 18 & 42,9 & 5,79 & 33,0 & 4,5 & 31,8 \\
4 & Miyagi & 3 & 21 & 39,8 & 4,27 & 31,5 & 4,5 & 31,7 \\
5 & Miyagi & 5 & 3 & 35,0 & 3,57 & 30,8 & 4,3 & 31,5 \\
6 & Miyagi & 3 & 18 & 39,3 & 4,13 & 31,4 & 4,3 & 31,5 \\
7 & Nara & 4 & 13 & 39,7 & 4,45 & 31,7 & 4,3 & 31,5 \\
8 & Nara & 2 & 15 & 40,5 & 5,02 & 32,3 & 4,4 & 31,6 \\
9 & Miyagi & 1 & 3 & 32,1 & 1,85 & 29,1 & 4,1 & 31,4 \\
10 & Miyagi & 5 & 16 & 32,7 & 2,95 & 30,2 & 4,0 & 31,2 \\
\hline
\end{tabular}

Além disso, será resgatado material vegetativo dos 10 melhores indivíduos para multiplicação assexuada e posterior instalação de testes clonais em diferentes condições, com o intuito de confirmar a superioridade existente no material genético. Segundo Golle et al. (2009), a seleção de árvores-elite e a sua propagação por meio de clonagem é um dos métodos mais utilizados no melhoramento florestal.

Resende e Barbosa (2005) recomendam incluir na seleção de clones todos os indivíduos com valores genotípicos preditos (g) superiores à média populacional. No presente estudo, foram selecionados apenas 10 indivíduos das duas procedências com maiores valores genotípicos preditos, objetivando maiores ganhos de produtividade em função do maior grau de seleção. 
Embora haja relato de enraizamento dessa espécie de até 87\% (JULL et al., 1994), Kratz et al. (2011) verificaram o enraizamento de apenas $23 \%$ das estacas, mesmo com a utilização de ácido indolbutírico a $4.000 \mathrm{mg} . \mathrm{L}^{-1}$ de concentração. Dessa forma, maiores estudos relacionados à propagação vegetativa dessa espécie são necessários.

A seleção dos indivíduos para clonagem foi realizada através do ordenamento efetuado pelo software SELEGEN (RESENDE, 2002b) para a variável adimensional (DAP x Sobrevivência). Entretanto, os valores utilizados para expressar as características genéticas dos indivíduos são os resultados obtidos com a variável DAP.

Takata e Teraoka (2002), estudando plantios de $C$. japonica no Japão, concluíram que muitas características fundamentais da qualidade da madeira diferem significativamente entre cultivares, sugerindo um forte controle genético dessas características. Em função disso, recomendam-se maiores estudos tecnológicos da madeira do material selecionado, para complementação do programa de melhoramento ora proposto.

\section{CONCLUSÕES}

- O potencial produtivo das procedências japonesas de C. japonica testadas foi confirmado. As procedências Miyagi e Nara merecem destaque, uma vez que apresentaram boa sobrevivência e crescimento volumétrico superior a $20 \mathrm{~m}^{3} \cdot \mathrm{ha}^{-1} \cdot \mathrm{ano}^{-1}$.

- A estratégia de melhoramento proposta prevê a formação de uma Área de Produção de Sementes, com ganho de crescimento diamétrico de 9\%, e um Pomar Clonal de Sementes, com ganho de 14\%.

\section{AGRADECIMENTOS}

Ao administrador da Estação Experimental de Rio Negro, Prof. Dr. Carlos Firkowski, e aos funcionários Eng. Agr. Gilson Machado Rosa e Osmiro Valério Neto, pela colaboração durante a coleta de dados e instalação da Área de Produção de Sementes. Ao Prof. Dr. Rudi Arno Seitz (in memoriam), pela guarda e monitoramento do experimento durante muitos dos 31 anos.

\section{REFERÊNCIAS}

ALMEIDA, M. H.; FARIA, C.; BELERIQUE, J.; NOBREGA, C.; PENACHO, L.; ROCHETA, M. Resultados preliminares dos testes genéticos com Cryptomeria japonica na Região Autónoma dos Açores. In: CONGRESSO FLORESTAL NACIONAL: A FLORESTA E AS GENTES, 5., 2005, Viseu. Anais... Viseu, 2005.

ALVES, S. T.; SHIMIZU, J. Y.; HIGA, A. R.; HIGA, R. C. V. Teste de procedência de Cryptomeria japonica em três regiões do Estado do Paraná. Curitiba: Embrapa CNPF, 1984. 3 p. (EMBRAPAURPFS. Pesquisa em andamento, n. 40).

CARPANEZZI, A.; CARVALHO, P. E. R. Zoneamento ecológico para plantios florestais do estado de Santa Catarina. Curitiba: EMBRAPA-CNPF, 1988. 103 p. (EMBRAPA-CNPF. Documento 21).

CARVALHO, P. E. R. Novas alternativas para reflorestamento. Revista da Madeira, Curitiba, v. 11, n. 60, p. $66-67,2001$.

EMPRESA BRASILEIRA DE PESQUISA AGROPECUÁRIA, Centro Nacional de Pesquisas Florestais - CNPF. Zoneamento ecológico para plantios florestais no estado do Paraná, Curitiba: EMBRAPA, 89 p., 1986.

EMPRESA BRASILEIRA DE PESQUISA AGROPECUÁRIA, Centro Nacional de Pesquisas Florestais - CNPF. Zoneamento ecológico para plantios florestais no estado de Santa Catarina, Curitiba: EMBRAPA, 113 p., 1988.

FABIÃO, A. M. D. Árvores e florestas. Portugal: Publicações Europa-América, 1987. 228 p. 
GÉRARD, J.; BEAUCHÊNE, J.; FOUQUET, D.; GUIBAL, D.; LANGBOUR, P.; THEVENON, M. F.; THIBAUT, A.;VERNAY, M. TROPIX 5.0: Caractéristiques technologiques de 215 essences tropicales. Montpellier: CIRAD-Forêt, 2003. Disponível em: <http://www.cirad.fr/activities/bois/ew/syst/asia/ sugi.pdf>. Acesso em: 28/03/2011.

GOLLE, D. P.; REINIGER, L. R. S.; CURTI, A. R.; BEVILACQUA, C. B. Melhoramento Florestal: ênfase na aplicação da biotecnologia. Ciência Rural, Santa Maria, v. 39, n. 5, p. 1606 - 1613, 2009.

GURGEL FILHO, O. A. O comportamento florestal das coníferas exóticas. Silvicultura em São Paulo, São Paulo, v. 3, n. 3, p. 129 - 188, 1964.

KRATZ, D.; WENDLING, I.; BRONDANI, G. E. Concentrações de ácido indolbutírico no enraizamento de Cryptomeira japonica. Journal of Biotechnology and Biodiversity, v. 2, n. 3, p. 1 - 8, 2011.

KURINOBU, S.; CHIGIRA, O. Second-generation plus tree selection by local foresters in controlled pollinated progenies of the first-generation plus trees of Sugi (Cryptomeria japonica) - estimates of genetic parameters on growth and form traits in three genetic tests at 30 years of age. Boulletin of the National Forest Tree Breeding Center, Japan, n. 17, p. 177 - 188, 2000.

JULL, L. G.; WARREN, S. L.; BLAZICH, F. A. (1994). Rooting Yoshino Cryptomeria stem cuttings as influenced by grow stage, branch order, and IBA treatment. HortScience, v. 29, p. 1532 - 1535.

LERNER, I. M. The genetic basis of selection. New York: John Wiley and Sons, 1977. 298 p.

OHBA, K.; WATANABE, M.; NOGUCHI, T.; MOMOSE, Y.; KAWANO, K.; YOKOYAMA, S.; OOTU, M.; MURAI, M. Variation on height growth and its heritability of F1 seedlings derived from crosses among Sugi cultivars propagated by cuttings and Sugi individual, Cryptomeria japonica D. Don. Journal of the Japanese Forestry Society, v. 52, n. 2, p. 51 - 57, 1970.

OHBA, K. Genetic structure of geographical marginal populations of Cryptomeria japonica. Canadian Juornal of Forest Research, v. 23, p. 859 - 863, 1993.

PEREIRA, J. C. D.; HIGA, R. C. V.; SHIMIZU, J. Y. Propriedades da madeira do cedrinho japonês. Colombo: Embrapa Florestas, 2003. 4 p. (Comunicado técnico, n. 88).

RESENDE, M. D. V.; HIGA, A. R. Estimação de valores genéticos no melhoramento de Eucalyptus: seleção em um caráter com base em informações do indivíduo e de seus parentes. Boletim de Pesquisa Florestal, Colombo, n.28/29, p. 11 - 36, 1994.

RESENDE, M. D. V. Genética biométrica e estatística no melhoramento de plantas perenes. EMBRAPA, Informação Tecnológica, Brasília, 975 p., 2002a.

RESENDE, M. D. V. Software SELEGEN-REML/BLUP. EMBRAPA, Colombo, Novembro, 2002b.

RESENDE, M. D. V.; BARBOSA, M. H. P. Melhoramento genético de plantas com propagação assexuada. Colombo: Empresa Brasileira de Pesquisa Agropecuária, 2005. 130 p.

SAKAI, K-I; MUKAIDE, H. Estimation of genetic environmental and competitional variances in standing forests. Silvae Genetica, v. 16, n. 5 - 6, p. 149 - 152, 1966.

SANTOS, C. B.; LONGHI, S. J.; HOPPE, J. M.; MOSCOVICH, S. A. Efeito do volume de tubetes e tipos de substratos na qualidade de mudas de Cryptomeria japonica (L.F.) D. Don. Ciência Florestal, Santa Maria, v. 10, n. 2, p. 1 - 15, 2000.

SEBBENN, A. M. Sistema de reprodução em espécies arbóreas tropicais e suas implicações para a seleção de árvores matrizes para reflorestamentos ambientais. In: HIGA, A. R.; SILVA, L. D. (Coords.) Pomar de Sementes de Espécies Florestais Nativas. Curitiba: FUPEF, 2006. p. 93 - 138.

SEBBENN, A. M.; PIRES, C. L. G.; STORCK, L.; CUSTÓDIO FILHO, A.; ROSA, P. R. F. Variação genética em progênies de meio-irmãos de Pinus caribaea Mor. var. bahamensis Bar. et Gol. na região de Bebedouro-SP. Rev. Inst. Flor., São Paulo, v. 6, p. 63 - 73. 1994. 
SHIMIZU, J. Y.; MAIOCHI, R. A. Criptoméria como espécie alternativa para produção de madeira no Paraná. Pesquisa Florestal Brasileira, Colombo, n. 54, p. 63 - 70, 2007.

SHYUR, L. F., HUANG, C. C.; LO, C. P.; CHIU, C. Y; CHEN, Y. P.; WANG, S. Y.; CHANG, S. T. Hepatoprotective phytocompounds from Cryptomeria japonica are potent modulators of inflammatory mediators. Phytochemistry n. 69. p. 1348 - 1358, 2008.

TAKATA, K.; TERAOKA, Y. Genotypic effects on the variation of wood quality and growth traits in plantation forest made by cutting cultivars of Japanese Cedar. Journal of Wood Science, v. 48, p. 106 $113,2002$.

VENCOVSKY. R. Herança quantitativa. In: PATENIANI, E. Melhoramento e produção do milho no Brasil. Piracicaba, 1978. p. 122-210.

YAMASHITA, T.; KASUYA, N.; NISHIMURA, S.; TAKEDA, H. Comparison of two coniferous plantation in central Japan with respect to forest productivity, growth phenology and soil nitrogen dynamics. Forest Ecology and Management, n. 200, p. 215 - 226, 2004.

ZHU, J.; TADOOKA, N.; TAKATA, K.; KOIZUMI, A. Growth and wood quality of Sugi (Cryptomeria japonica) planted in Akita prefecture (II). Juvenile/mature wood determination of aged trees. Journal of Wood Science, n. 51, p. 95 - 101, 2005. 